\title{
On Planar Five-bar Motion Generation with a Driver Torque Constraint"
}

\author{
Qiong SHEN**, Yahia M. Al-SMADI***, Kevin RUSSELL**** \\ and Raj S. SODHI** \\ ** Department of Mechanical Engineering, New Jersey Institute of Technology \\ University Heights, Newark, NJ07102-1982, U.S.A. \\ *** Parsons Transportation Group \\ New York, NY 10005, U.S.A. \\ **** Armaments Engineering and Technology Center, \\ U.S. Army Research Development and Engineering Center, \\ Picatinny, NJ 07806-5000, U.S.A. \\ E-mail: kevin.russell1@us.army.mil
}

\begin{abstract}
In motion generation, the objective is to calculate the mechanism parameters required to achieve or approximate a set of prescribed rigid-body positions. This work introduces a new design constraint that considers driving link static torque for a given rigid-body load. By incorporating this new constraint into a conventional planar five-bar motion generation model, planar five-bar mechanisms are synthesized to not only achieve prescribed rigid-body positions, but also satisfy maximum driver torque for a given rigid-body load.
\end{abstract}

Key words: Motion Generation, Planar Five-Bar, Rigid-Body, Static Torque

\section{Introduction}

\subsection{Motion generation}

In motion generation, the objective is to calculate the mechanism parameters required to achieve or approximate a set of prescribed rigid-body positions. This mechanism design objective is particularly useful when the rigid-body must achieve a specific displacement sequence for effective operation (e.g., specific tool paths and orientations for accurate fabrication operations). In Figure 1, four prescribed rigid-body positions are defined by the $\mathrm{x}$ and $\mathbf{y}$-coordinates of variables $\mathbf{p}, \mathbf{q}$ and $\mathbf{r}$ and the calculated mechanism parameters are the $\mathrm{x}$ and y-coordinates of fixed pivot variables $\mathbf{a}_{0}$ and $\mathbf{b}_{0}$ and moving pivot variables $\mathbf{a}_{1}, \mathbf{b}_{1}$ and $\mathbf{c}_{1}$.

Motion generation for planar five-bar mechanisms is a well-established field. Recent contributions include the works of Musa, Russell and Sodhi ${ }^{(1)(2)}$ that consider motion generation of adjustable planar five-bar motion generators with prescribed rigid-body positions and rigid-body positions with tolerances. The works of Balli and Chand ${ }^{(3)(4)}$ introduce a complex number method for the synthesis of a planar five-bar motion generator with prescribed timing and a method to synthesize a planar five-bar mechanism of variable topology type with transmission angle contol. Nokleby and Podhorodeski ${ }^{(5)}$ presented an optimization method to synthesize Grashof five-bar mechanisms. Wang and Yan ${ }^{(6)}$ presented an approach for synthesizing planar five-bar linkages with five prescribed precision positions. Basu and Farhang ${ }^{(7)}$ introduced a mathematical formulation for the approximate analysis and design of two-input, small-crank five-bar mechanisms for function generation. Dou and Ting ${ }^{(8)}$ introduced a method to identify to rotatability and branch condition in linkages containing simple geared five-bar chains. Lin and Chaing ${ }^{(9)}$ extended pole method 
for use in the synthesis planar, geared five-bar function generators. Ge and Chen (10) introduced a software-based approach for the atlas method on path synthesis of geared five-bar mechanisms. Ge and Chen also studied the effect of link length, crank angles and gear tooth ratio on the motion of the geared five-bar linkage ${ }^{(11)}$. Li and Dao ${ }^{(12)}$ introduced a complex number method for the synthesis for geared, five-bar guidance mechanisms. Huang and Roth ${ }^{(13)}$ considered static force conditions as well as motions in the dimensional synthesis of planar and spatial linkages,

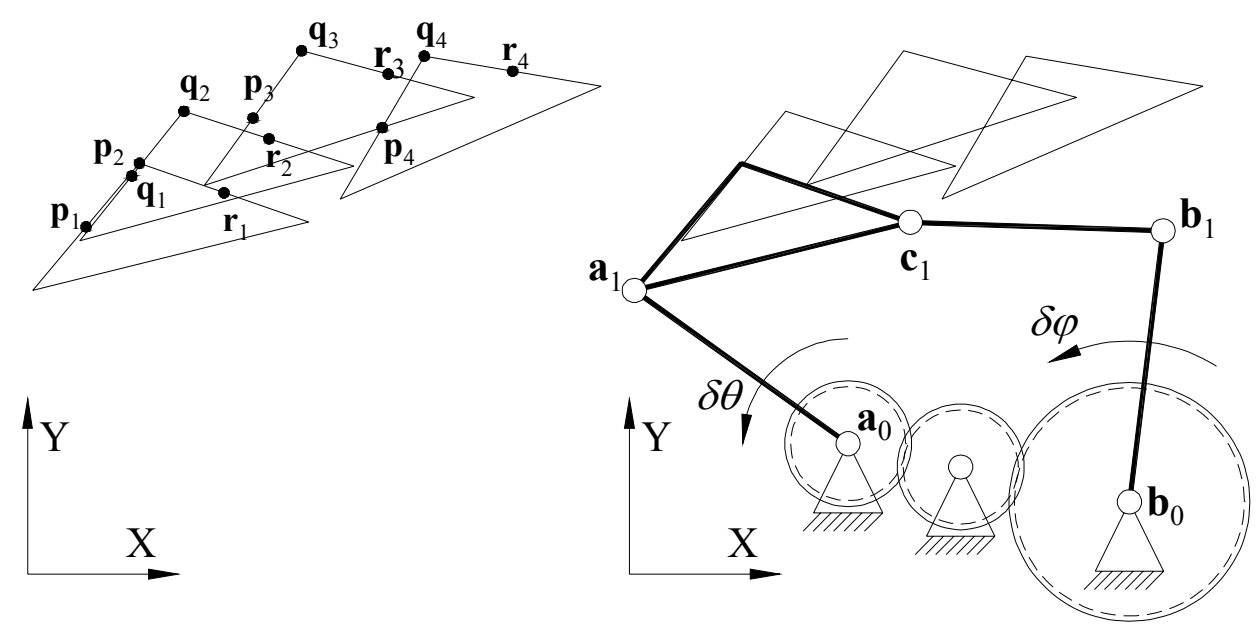

Figure 1. Prescribed rigid-body positions and calculated planar five-bar mechanism

\subsection{Motivation and scope of work}

Using conventional motion generation methods the user can only calculate the mechanism parameters required to achieve or approximate a set of prescribed rigid-body positions. Although such solutions are useful for preliminary kinematic analyses, other factors (e.g., static loads, dynamic loads, stresses, strains, etc.) must be considered prior to fabricating a physical prototype of the mechanical design. This work considers static driving link torque given the load of the rigid-body. By incorporating this new constraint into a conventional planar five-bar motion generation model, planar five-bar mechanisms are synthesized to not only achieve prescribed rigid-body positions, but also satisfy maximum driver torque for a given rigid-body load.

\section{Planar five-bar motion generation and static torque constraint}

\subsection{Conventional planar five-bar motion generation}

Equations (1) through (3) encompass a conventional planar five-bar motion generation model $^{(1)(2)(14)(15)}$.

$$
\begin{aligned}
& \left(\left[\mathbf{D}_{1 j}\right] \mathbf{a}_{1}-\mathbf{a}_{0}\right)^{T}\left(\left[\mathbf{D}_{1 j}\right] \mathbf{a}_{1}-\mathbf{a}_{0}\right)-L_{1}{ }^{2}=0, \mathrm{j}=1,2,3,4 \\
& \left(\left[\mathbf{D}(\delta \varphi)_{1 j}\right] \mathbf{b}_{1}-\mathbf{b}_{0}\right)^{T}\left(\left[\mathbf{D}(\delta \varphi)_{1 j}\right] \mathbf{b}_{1}-\mathbf{b}_{0}\right)-L_{2}{ }^{2}=0, \mathbf{j}=1,2,3,4 \\
& \left(\left[\mathbf{D}_{1 j}\right] \mathbf{c}_{1}-\left[\mathbf{D}(\delta \varphi)_{1 j}\right] \mathbf{b}_{1}\right)^{T}\left(\left[\mathbf{D}_{1 j}\right] \mathbf{c}_{1}-\left[\mathbf{D}(\delta \varphi)_{1 j}\right] \mathbf{b}_{1}\right)-L_{3}{ }^{2}=0, \mathbf{j}=1,2,3,4
\end{aligned}
$$

These equations are "constant length" constraints and ensure the fixed lengths of links $\mathbf{a}_{0}-\mathbf{a}_{1}, \mathbf{b}_{0}-\mathbf{b}_{1}$ and $\mathbf{b}_{1}-\mathbf{c}_{1}$ throughout the prescribed rigid-body displacements. Variables $L_{l}, L_{2}$ and $L_{3}$ in Equations (1) through (3) are the prescribed scalar lengths of links $\mathbf{a}_{0}-\mathbf{a}_{1}, \mathbf{b}_{0}-\mathbf{b}_{1}$ and $\mathbf{b}_{1}-\mathbf{c}_{1}$ respectively. 


$$
\begin{gathered}
{\left[\mathbf{D}_{1 j}\right]=\left[\begin{array}{ccc}
p_{j x} & q_{j x} & r_{j x} \\
p_{j y} & q_{j y} & r_{j y} \\
1 & 1 & 1
\end{array}\right]\left[\begin{array}{ccc}
p_{l x} & q_{l x} & r_{l x} \\
p_{l y} & q_{l y} & r_{l y} \\
1 & 1 & 1
\end{array}\right]^{-1}, \mathrm{j}=1,2,3,4} \\
{\left[\mathbf{D}(\delta \varphi)_{1 j}\right]=\left[\begin{array}{ccc}
\cos (\delta \varphi)_{1 j} & -\sin (\delta \varphi)_{1 j} & -b_{0 x} \cos (\delta \phi)_{1 j}+b_{0 y} \sin (\delta \phi)_{1 j}+b_{0 x} \\
\sin (\delta \varphi)_{1 j} & \cos (\delta \varphi)_{1 j} & -b_{0 x} \sin (\delta \phi)_{1 j}-b_{0 y} \cos (\delta \phi)_{1 j}+b_{0 y} \\
0 & 0 & 1
\end{array}\right],}
\end{gathered}
$$

$\mathrm{j}=1,2,3,4$

Equation (4) is a rigid-body planar displacement matrix. Equation (5) is the angular displacement matrix for link $\mathbf{b}_{0}-\mathbf{b}_{1}$ where

$$
\begin{aligned}
& M_{1 j}=\left[\begin{array}{c:c:c}
1 & & \\
1 & \mathbf{a}_{1}-\mathbf{a}_{0} & {\left[D_{1 j}\right] \mathbf{a}_{1}-\mathbf{a}_{0}} \\
1 & 0 & 0
\end{array}\right] \\
& \cos (\delta \theta)_{1 j}=\frac{\left(\mathbf{a}_{1}-\mathbf{a}_{0}\right) \cdot\left(\left[D_{1 j}\right] \mathbf{a}_{1}-\mathbf{a}_{0}\right)}{\left|\mathbf{a}_{1}-\mathbf{a}_{0}\right| \cdot\left|\left[D_{1 j}\right] \mathbf{a}_{1}-\mathbf{a}_{0}\right|}, \sin (\delta \theta)_{1 j}=\frac{\operatorname{det}\left[M_{1 j}\right]}{\left|\mathbf{a}_{1}-\mathbf{a}_{0}\right| \cdot\left|\left[D_{1 j}\right] \mathbf{a}_{1}-\mathbf{a}_{0}\right|} \\
& (\delta \theta)_{1 j}=\arctan 2\left(\sin (\delta \theta)_{1 j}, \cos (\delta \theta)_{1 j}\right)
\end{aligned}
$$

and $(\delta \varphi)_{1 \mathrm{j}}=k(\delta \theta)_{1 \mathrm{j}}$. Variable $k$ represents the gear ratio of the gear train joining grounded links $\mathbf{a}_{0}-\mathbf{a}_{1}$ and $\mathbf{b}_{0}-\mathbf{b}_{1}$. From this conventional planar five-bar motion generator model, 12 of the 13 unknown variables $\mathbf{a}_{0}, \mathbf{a}_{1} L_{1}, \mathbf{b}_{0}, \mathbf{b}_{1}, L_{2}, \mathbf{c}_{1}$, and $L_{3}$ are calculated with one arbitrary choice of parameter (where $\mathbf{a}_{0}=\left[a_{0 x}, a_{0 y}, 1\right], \mathbf{a}_{1}=\left[a_{1 x}, a_{1 y}, 1\right], \mathbf{b}_{0}=\left[b_{0 x}, b_{0 y}, 1\right], \mathbf{b}_{1}=\left[b_{1 x}, b_{1 y}, 1\right]$ and $\left.\mathbf{c}_{1}=\left[c_{l x}, c_{l y}, 1\right]\right)$.

\subsection{Driver link static torque}

With an external or body load $\mathbf{W}$ acting on the rigid-body of the planar five-bar mechanism, a driving link torque $\mathbf{T}$ achieves static equilibrium. In Figure 2, the load $\mathbf{W}$ is applied at the arbitrary rigid-body point $\mathbf{q}$. To formulate the driver link static torque constraint, the moment condition $\Sigma \mathbf{M}=0$ is considered about the fixed pivot $\mathbf{a}_{0}$. As illustrated in Figure 3, the fixed pivot reaction loads $\mathbf{R}_{\mathrm{a} 0}$ and $\mathbf{R}_{c 1}$ are considered in the moment condition. The resulting equilibrium equation of the moments about the fixed pivot $\mathbf{a}_{0}$ is (notice that link $\mathbf{b}_{1}-\mathbf{c}_{1}$ is a two-force member)

$$
\overrightarrow{a_{0} c_{1}} \times \mathbf{R}{ }_{c 1}+\overrightarrow{a_{0} q} \times \mathbf{W}+\mathbf{T}_{a}=0
$$

where

$$
\mathbf{R}_{c 1}=R_{c} \frac{\overrightarrow{b_{1} c_{1}}}{\left|\overrightarrow{b_{1} c_{1}}\right|}
$$

and the reaction load $R_{c}$ is a real number that varies with the mechanism driver position.

By expanding the vectors $\overrightarrow{a_{0} c_{1}}$ and $\overrightarrow{a_{0} q}$, Equation (6) becomes

$$
\left(\overrightarrow{a_{0} a_{1}}+\overrightarrow{a_{1} c_{1}}\right) \times \mathbf{R}_{c 1}+\left(\overrightarrow{a_{0} a_{1}}+\overrightarrow{a_{1} q}\right) \times \mathbf{W}+\mathbf{T}_{a}=0
$$

Next, the moment condition $\Sigma \mathbf{M}=0$ is considered about the moving pivot $\mathbf{a}_{1}$ considering all of the rigid-body loads as illustrated in Figure $4 \mathrm{a}$. The resulting equilibrium equation of the moments about the moving pivot $\mathbf{a}_{1}$ is

$$
\overrightarrow{a_{1} c_{1}} \times \mathbf{R}_{c 1}+\overrightarrow{a_{1} q} \times \mathbf{W}=0
$$

Substituting Equation (9) into Equation (8) produces

$$
\overrightarrow{a_{0} a_{1}} \times \mathbf{R}_{c 1}+\overrightarrow{a_{0} a_{1}} \times \mathbf{W}+\mathbf{T}_{a}=0
$$

Substituting Equation (7) into Equations (9) and (10) produces 


$$
\begin{aligned}
& \frac{R_{c}}{\left|\overrightarrow{b_{1} c_{1}}\right|} \overrightarrow{a_{1} c_{1}} \times \overrightarrow{b_{1} c_{1}}=\mathbf{W} \times \overrightarrow{a_{1} q} \\
& \frac{R_{c}}{\left|\overrightarrow{b_{1} c_{1}}\right|} \overrightarrow{a_{0} a_{1}} \times \overrightarrow{b_{1} c_{1}}=\mathbf{W} \times \overrightarrow{a_{0} a_{1}}-\mathbf{T}_{a}
\end{aligned}
$$

Combining Equations (11) and (12) produces

$$
\mathbf{T}_{a}=\left(\frac{\left(\mathbf{W} \times \overrightarrow{a_{1} q}\right)_{3}}{\left(\overrightarrow{a_{1} c_{1}} \times \overrightarrow{b_{1} c_{1}}\right)_{3}} \overrightarrow{b_{1} c_{1}}+\mathbf{W}\right) \times \overrightarrow{a_{0} a_{1}}
$$

where

$\mathbf{W}=\left[\begin{array}{lll}0 & -w & 0\end{array}\right]^{T}, \quad \mathbf{T}_{\mathbf{a}}=\left[\begin{array}{lll}0 & 0 & \tau_{a j}\end{array}\right]^{T}, \overrightarrow{a_{1} q}=\mathbf{q}_{j}-\left[\mathbf{D}_{1 j}\right] \mathbf{a}_{1}, \overrightarrow{a_{0} a_{1}}=\left[\mathbf{D}_{1 j}\right] \mathbf{a}_{1}-\mathbf{a}_{0}$, $\overrightarrow{b_{1} c_{1}}=\left[\mathbf{D}_{1 j}\right] \mathbf{c}_{1}-\left[\mathbf{D}(\delta \phi)_{1 j}\right] \mathbf{b}_{1}$ and $\overrightarrow{a_{1} c_{1}}=\left[\mathbf{D}_{1 j}\right] \mathbf{c}_{1}-\left[\mathbf{D}_{1 j}\right] \mathbf{a}_{1}$. The terms $\left(\mathbf{W} \times \overrightarrow{\mathrm{a}_{1} \mathrm{q}}\right)_{3}$ and $\left(\overrightarrow{\mathrm{a}_{1} \mathrm{c}_{1}} \times \overrightarrow{\mathrm{b}_{1} \mathrm{c}_{1}}\right)_{3}$ are the third elements of the corresponding vectors.

Finally, the moment condition $\Sigma \mathbf{M}=0$ is considered about the fixed pivot $\mathbf{b}_{0}$ for link $\mathbf{b}_{0}-\mathbf{b}_{1}$ as illustrated in Figure $4 \mathrm{~b}$. The resulting equilibrium equation of the moments about the fixed pivot $\mathbf{b}_{0}$ is

$$
\mathbf{T}_{b}-\overrightarrow{b_{0} b_{1}} \times \mathbf{R}_{c 1}=0
$$

Substituting Equation (11) into Equation (14) gives

$$
\mathbf{T}_{b}=\frac{\left(\mathbf{W} \times \overrightarrow{a_{1} q}\right)_{3}}{\left(\overrightarrow{a_{1} c_{1}} \times \overrightarrow{b_{1} c_{1}}\right)_{3}} \overrightarrow{b_{0} b_{1}} \times \overrightarrow{b_{1} c_{1}}
$$

When the two grounded links of the five-bar mechanism are connected by a gear train as illustrated in Figure 1, the rotational displacements of the two grounded links have the functional relationship $\delta \varphi=f(\delta \theta)=k \delta \theta$ where the constant " $k$ " represents the gear ratio. Assuming the power loss in the train is negligible, the equivalent torque about $\mathbf{a}_{0}$ due to $\mathbf{T}_{\mathrm{b}}$ is

$$
\mathbf{T}_{b}^{\prime}=k \mathbf{T}_{b}
$$

The total torque about $\mathbf{a}_{0}$ required for static equilibrium is therefore

$$
\mathbf{T}=\mathbf{T}_{a}+\mathbf{T}_{b}^{\prime}=\mathbf{T}_{a}+k \mathbf{T}_{b}=\frac{\left(\mathbf{W} \times \overrightarrow{a_{1} q}\right)_{3}}{\left(\overrightarrow{a_{1} c_{1}} \times \overrightarrow{b_{1} c_{1}}\right)_{3}}\left(\overrightarrow{b_{1} c_{1}} \times \overrightarrow{a_{0} a_{1}}+k \cdot \overrightarrow{b_{0} b_{1}} \times \overrightarrow{b_{1} c_{1}}\right)+\mathbf{W} \times \overrightarrow{a_{0} a_{1}}
$$

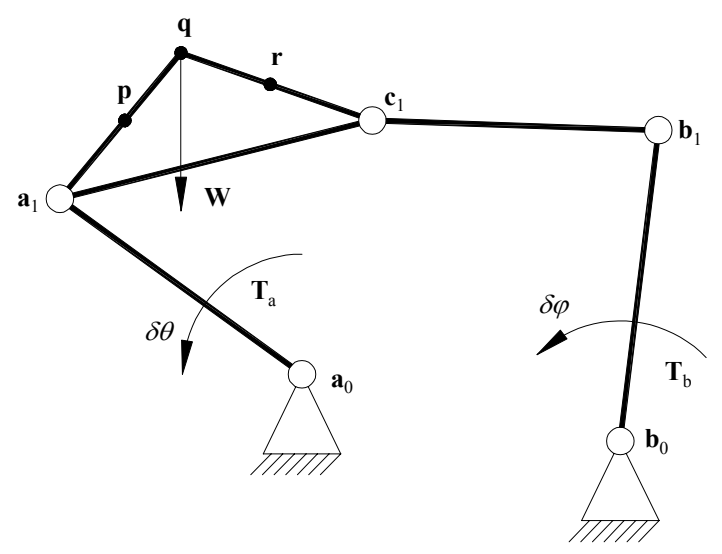

Figure 2. Planar five-bar mechanism in static equilibrium 
$\mathbf{a}_{1}$

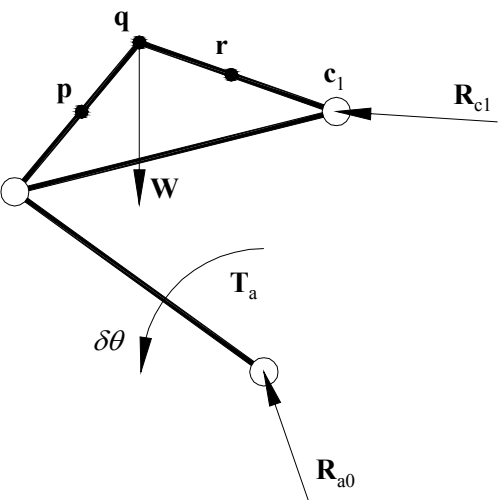

Figure 3. Planar five-bar mechanism in static equilibrium with reaction loads $\mathbf{R}_{\mathrm{a} 0}$ and $\mathbf{R}_{\mathrm{c} 1}$

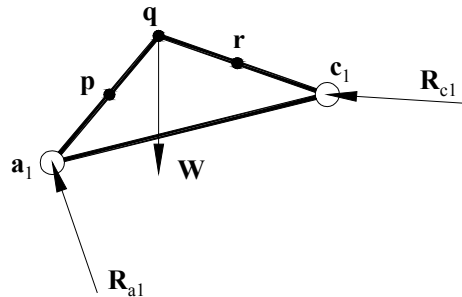

(a) rigid-body

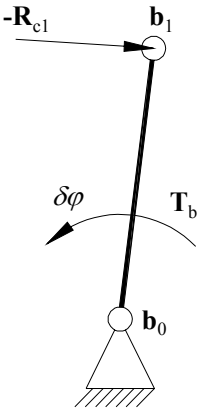

(b) link $\mathbf{b}_{0} \mathbf{b}_{1}$

Figure 4. Planar five-bar mechanism rigid-body (a) and link $\mathbf{b}_{0} \mathbf{b}_{1}$ (b) in static equilibrium

2.3 Planar five-bar motion generation exterior penalty function with driver static torque constraint

When Equation (17) is incorporated into the motion generation model in Section 2.1, the resulting model is useful for calculating the fixed and moving pivot parameters required to approximate a set of prescribed rigid-body positions and satisfy driver static torque requirements.

Formulating Equations (1) through (3) into a single objective function to be minimized yields

$$
\begin{aligned}
f(\mathbf{X})=\sum_{j=1}^{4}\{ & {\left[\left(\left[\mathbf{D}_{1 j}\right] \mathbf{a}_{1}-\mathbf{a}_{0}\right)^{T}\left(\left[\mathbf{D}_{1 j}\right] \mathbf{a}_{1}-\mathbf{a}_{0}\right)-L_{i}^{2}\right]^{2} } \\
& +\left[\left(\left[\mathbf{D}(\delta \phi)_{1 j}\right] \mathbf{b}_{1}-\mathbf{b}_{0}\right)^{T}\left(\left[\mathbf{D}(\delta \phi)_{1 j}\right] \mathbf{b}_{1}-\mathbf{b}_{0}\right)-L_{2}^{2}\right]^{2} \\
& \left.+\left[\left(\left[\mathbf{D}_{1 j}\right] \mathbf{c}_{1}-\left[\mathbf{D}(\delta \phi)_{1 j}\right] \mathbf{b}_{1}\right)^{T}\left(\left[\mathbf{D}_{1 j}\right] \mathbf{c}_{1}-\left[\mathbf{D}(\delta \phi)_{1 j}\right] \mathbf{b}_{1}\right)-L_{3}^{2}\right]^{2}\right\}
\end{aligned}
$$

where $\mathbf{X}=\left(a_{0 x}, a_{0 y}, a_{l x}, a_{l y}, b_{0 x}, b_{0 y}, b_{l x}, b_{l y}, c_{l x}, c_{l y}, L_{l}, L_{2}, L_{3}\right)^{T}$

In practice, both the magnitude and direction of the driver static torque could be constrained. These two constraints can be expressed as

$$
g_{j}(\mathbf{X})=\left(\mathbf{T}_{j}\right)_{3}\left[\left(\mathbf{T}_{j}\right)_{3}-\tau_{\max }\right] \leq 0, \quad j=1,2,3,4
$$

where the subscript " 3 " stands for the third element of the corresponding vectors. Substituting Equation (17) into Equation (19) gives a non-linear inequality constraint in standard form which will keep the driving torque counterclockwise and its magnitude less 
than $\tau_{\max }$. To keep the driving torque clockwise and its magnitude less than $\tau_{\max }$ (as in the forthcoming example), the constraint inequality should be

$$
g_{j}(\mathbf{X})=\left(\mathbf{T}_{j}\right)_{3}\left[\left(\mathbf{T}_{j}\right)_{3}+\tau_{\max }\right] \leq 0, \quad j=1,2,3,4
$$

The constrained minimization problem can be converted into an artificial unconstrained problem using the exterior penalty function. The modified objective function $P(\mathbf{X})$ is defined as

$$
P(\mathbf{X})=f(\mathbf{X})+\frac{1}{r} \sum_{j=1}^{4}\left\{\max \left[0, g_{j}(\mathbf{X})\right]\right\}^{2}
$$

where $1 / \mathrm{r}$ is referred to as penalty number (where $0<\mathrm{r} \leq 1.0)^{(14)}$. If an inequality constraint is satisfied, then $\mathrm{g}_{\mathrm{j}} \leq 0$ and $\max \left[0, \mathrm{~g}_{\mathrm{j}}(\mathbf{X})\right]$ will return 0 and therefore that constraint will not contribute to $P(\mathbf{X})$. If an inequality constraint is violated (i.e. $\mathrm{g}_{\mathrm{j}}>0$ ), a large contribution will be added to $P(\mathbf{X})$. Minimizing $P(\mathbf{X})$ should eliminate the constraint violation. For successful objective function minimization, the penalty number $1 / \mathrm{r}$ must be very large. As $r$ decreases, $1 / \mathrm{r}$ increases and creates a series of suboptimal points that approach the constrained boundary from outside the feasible region ${ }^{(14)}$. Theoretically, the minimum $P(\mathbf{X})$ corresponds to the solution of the original problem only as $r \rightarrow 0$. In practice, a tolerance $\varepsilon$ is set as a stop criterion. Among various proposals for a rational basis of change in the value of $\mathrm{r}$ for successive minimizations is

$$
r=(\mathrm{FAC})^{1-\mathrm{IR}}
$$

where $I R=1$ initially and is increased by 1 after each successful suboptimum is found. A value of 10 is usually suggested for FAC ${ }^{(14)}$. The following is the pseudo code for the optimization $^{(16)}$ :

Step 1. Let $I R=1, \varepsilon>0, k=1$. Given initial $\mathbf{X}_{1}$ violating inequalities (19) or (20) (i.e. exterior point).

Step 2. Starting with $\mathbf{X}_{\mathrm{k}}$ solve problem (21) for $\mathbf{X}\left(\mathrm{r}_{\mathrm{k}}\right)$. Set $\mathbf{X}_{\mathrm{k}+1}=\mathbf{X}\left(\mathrm{r}_{\mathrm{k}}\right)$.

Step 3. If

$$
\frac{1}{r_{k}} \sum_{j=1}^{4}\left\{\max \left[0, g_{j}\left(\mathbf{X}_{k+1}\right)\right]\right\}^{2}<\varepsilon
$$

stop; otherwise $\mathrm{IR}_{\mathrm{k}+1}=\mathrm{IR}_{\mathrm{k}}+1, \mathrm{k}=\mathrm{k}+1$, go to step 2 .

In this work, a quasi-Newton method algorithm is used to solve problem (21).

\section{Example}

Table 1 includes the $\mathrm{x}$ and $\mathrm{y}$-coordinates (in feet) of four prescribed rigid-body positions. The prescribed weight for the rigid-body is 10 pounds and $k=1 / 2$. With initial guesses of $\mathbf{a}_{0}=(0,0), \mathbf{a}_{1}=(-0.5,1.1), L_{1}=1.2083, \mathbf{b}_{0}=(1,-0.4), \mathbf{b}_{1}=(1.5,0.2), L_{2}=0.7810, \mathbf{c}_{1}=(1$, $0.7), L_{3}=0.7071, \varepsilon=10^{-30}$, the required torques on the link $\mathrm{a}_{0} \mathrm{a}_{1}$ at the initial guessed position is $-5.3589 \mathrm{ft}-\mathrm{lbs}$ (which violets the inequality (19) used in computation for this example). The optimized results for $\tau_{\max }=12 \mathrm{ft}-\mathrm{lbs}$ are $\mathbf{a}_{0}=(-0.0855,0.0213), \mathbf{a}_{1}=(0.2477,1.1726)$, $L_{1}=1.1986, \mathbf{b}_{0}=(0.5378,0.0195), \mathbf{b}_{1}=(0.9744,0.4550), L_{2}=0.6167, \mathbf{c}_{1}=(0.4817,0.7192)$, $L_{3}=0.5591$. The achieved rigid-body positions and required torques on the crank (link $\left.\mathrm{a}_{0} \mathrm{a}_{1}\right)$ are listed in Table 2. The crank rotates clockwise 8.1906, 18.7362, and 29.8295 degrees respectively to achieve these positions. When the optimization stops, the $\mathrm{IR}=3$. Both achieved rigid-body positions and the driver static torques were validated by the SimMechanics module in MATLAB (top row in Figure 5).

Using the same initial guesses for $\mathbf{a}_{0}, \mathbf{a}_{1}, L_{1}, \mathbf{b}_{0}, \mathbf{b}_{1}, L_{2}$, and $\mathbf{c}_{1}, L_{3}$, and same inequality (19), the optimized results for $\tau_{\max }=8.5 \mathrm{ft}-\mathrm{lbs}$ are $\mathbf{a}_{0}=(0.1319,0.2492), \mathbf{a}_{1}=(0.0732,1.2750)$, $L_{1}=1.0275, \mathbf{b}_{0}=(0.5679,0.0768), \mathbf{b}_{1}=(0.9055,0.5110), L_{2}=0.5500, \mathbf{c}_{1}=(0.4694,0.7559)$, $L_{3}=0.5002$. The achieved rigid-body positions and required torques on crank (link $\left.\mathrm{a}_{0} \mathrm{a}_{1}\right)$ are listed in Table 3. The crank rotates clockwise 11.4459, 26.0789, and 41.5526 degrees 
respectively to achieve these positions. When the optimization stops, the $I R=3$. Both achieved rigid-body positions and the driver static torques were validated by the SimMechanics module in MATLAB (bottom row in Figure 5).

Table 1. Prescribed rigid-body positions ( $\mathrm{w}=10 \mathrm{lbs})$

\begin{tabular}{c|c|c|c}
\hline \hline \multicolumn{1}{c}{ p } & q & r \\
\hline \hline Pos 1 & $0.0536,1.0541$ & $0.2896,1.3620$ & $0.8139,1.2572$ \\
Pos 2 & $0.1857,1.0668$ & $0.5112,1.2778$ & $0.9701,1.0034$ \\
Pos 3 & $0.3745,1.0352$ & $0.7519,1.1250$ & $1.0923,0.7126$ \\
Pos 4 & $0.5720,0.9545$ & $0.9585,0.9207$ & $1.1516,0.4221$ \\
\hline
\end{tabular}

Table 2. Rigid-body positions achieved by synthesized planar five-bar mechanism $\left(\tau_{\max }=12 \mathrm{ft}-\mathrm{lbs}\right.$, $\mathrm{w}=10 \mathrm{lbs}$ )

\begin{tabular}{c|c|c|c|c}
\hline \hline \multicolumn{1}{c}{ p } & q & r & $\tau$ \\
\hline \hline Pos 1 & $0.0536,1.0541$ & $0.2896,1.3620$ & $0.8139,1.2572$ & 4.6036 \\
Pos 2 & $0.1857,1.0668$ & $0.5113,1.2778$ & $0.9701,1.0034$ & 7.0371 \\
Pos 3 & $0.3745,1.0352$ & $0.7519,1.1250$ & $1.0923,0.7127$ & 9.4818 \\
Pos 4 & $0.5720,0.9544$ & $0.9585,0.9207$ & $1.1516,0.4221$ & 11.5408 \\
\hline
\end{tabular}

Table 3. Rigid-body positions achieved by synthesized planar five-bar mechanism $\left(\tau_{\max }=8.5 \mathrm{ft}-1 \mathrm{bs}\right.$, $\mathrm{w}=10 \mathrm{lbs})$

\begin{tabular}{c|ccccc}
\hline \hline \multicolumn{1}{c}{ p } & q & r & $\tau$ \\
\hline \hline Pos 1 & $0.0536,1.0541$ & $0.2896,1.3620$ & $0.8139,1.2572$ & 4.3799 \\
Pos 2 & $0.1851,1.0648$ & $0.5110,1.2754$ & $0.9694,1.0003$ & 4.8535 \\
Pos 3 & $0.3754,1.0376$ & $0.7528,1.1271$ & $1.0930,0.7146$ & 6.7561 \\
Pos 4 & $0.5716,0.9536$ & $0.9581,0.9201$ & $1.1517,0.4217$ & 8.4924 \\
\hline
\end{tabular}



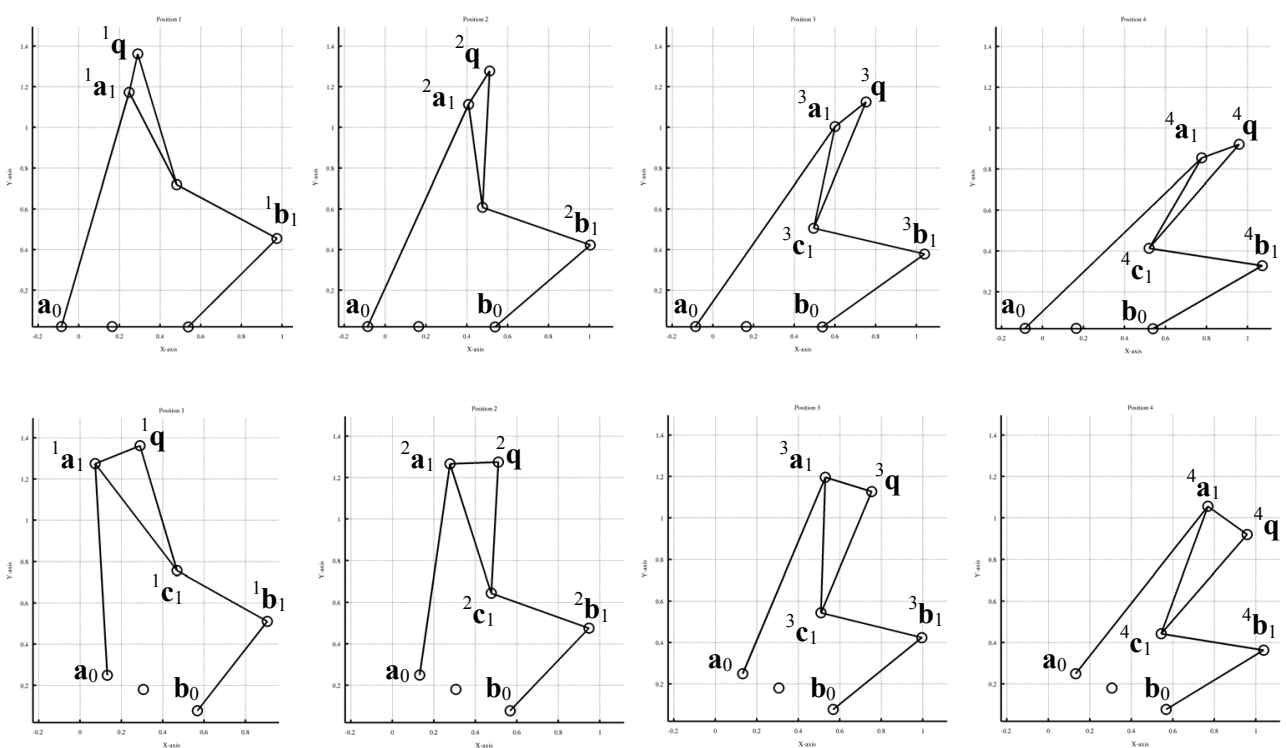

Figure 5. MATLAB plots of synthesized motion generator

\section{Discussion}

Equation (17) becomes invalid when the pivots $\mathbf{a}_{1}, \mathbf{b}_{1}$ and $\mathbf{c}_{1}$ are collinear. Such a state is possible when the five-bar mechanism reaches a "lock-up" or binding position. When pivots $\mathbf{a}_{1}, \mathbf{b}_{1}$ and $\mathbf{c}_{1}$ are collinear, the denominator in Equation (17) becomes zero (making the equation invalid). The calculated static driver torques-like the synthesized five-bar mechanism-correspond to only one mechanism branch. The Optimization Toolbox offered by mathematical analysis software MATLAB was used to codify and solve the problem (21) and the SimMechanics module in MATLAB was used to independently confirm the achieved rigid-body positions and driver static torques of the synthesized mechanisms. Although this work focuses on the formulation of a static torque constraint, static link deflection constraints-which will represent the mechanical system more accurately and completely-are forthcoming.

\section{Conclusion}

The driver link static torque constraint formulated in this work was demonstrated to be effective in calculating planar five-bar motion generator solutions that approximate the prescribed rigid-body positions and satisfy driver link static torque for a given rigid-body load. It was also demonstrated that the torque constraint could be used with the conventional planar five-bar motion generation model as a goal program and solved using a commercial goal program solver.

\section{References}

1. K. Russell and R. S. Sodhi, "Kinematic Synthesis of Adjustable Planar Five-Bar Mechanisms for Multi-Phase Motion Generation," JSME International Journal, Series C, Vol. 47, No. 1, pp. 345-349 (2004).

2. M. H. Musa, K. Russell and R. S. Sodhi, "Multi-Phase Motion Generation of Five-Bar Mechanisms with Prescribed Rigid-Body Tolerances," CSME Transactions, Vol. 30, No. 4, pp. 459-472 (2006).

3. S. S. Balli and S. Chand, "Five-Bar Motion and Path Generators with Variable Topology for Motion Between Extreme Positions," Mechanism and Machine Theory, Vol. 37, No. 11, pp. 1435-1445 (2002). 
4. S. S. Balli and S. Chand, "Synthesis of a Five-Bar Mechanism for Variable Topology Type with Transmission Angle Control," Journal of Mechanical Design, Vol. 126, No. 1, pp. 128-134 (2004).

5. S. B. Nokleby and R. P. Podhorodeski, "Optimization-Based Synthesis of Grashof Geared Five-Bar Mechanisms," Journal of Mechanical Design, Vol. 123, No. 4, pp. 529-534 (2001).

6. A. Wang and H. Yan, "Rigid-Body Guidance of Planar Five-Bar Linkages for Five Precision Positions," Journal of the Chinese Society of Mechanical Engineers, Vol. 12, No. 2, pp. 159-165 (1991).

7. P. S. Basu and K. Farhang, "Kinematic Analysis and Design of Two-Input, Five-Bar Mechanisms Driven by Relatively Small Cranks," 22nd Biennial Mechanisms Conference, Scottsdale, Arizona (1992).

8. X. Dou and K. Ting, "Branch Identification in Geared Five-Bar Chains," Journal of Mechanical Design, Vol. 118, No. 3, pp. 384-389 (1996).

9. C. Lin and C. H. Chaing, "Synthesis of Planar and Spherical Geared Five-Bar Function Generators by the Pole Method," Mechanism and Machine Theory, Vol. 27, No. 2, pp. 131-141 (1992).

10. W. Ge and Z. Chen, "Study of Geared Five-Bar Curves Based on Computer Mechanism Simulation with Variable Parameters," Mechanical Science and Technology, Vol. 18, No. 3, pp. 435-437 (1999).

11. W. Ge and Z. Chen, "Application Oriented Study of Characteristics of Jointn Loci of Geared Five-Bar Linkage," Journal of Northwestern Polytechnical University, Vol. 15, No. 4, pp. 542-546 (1997).

12. T. Li and W. Dao, "Kinematic Synthesis of Geared Linkage Mechanism for Body Guidance with Input Timing," Mechanical Science and Technology, Vol. 17, No. 6, pp. 869-870 (1999).

13. C. Huang and B. Roth, "Dimensional Synthesis of Closed-Loop Linkages to Match Force and Position Specifications," Journal of Mechanical Design, Vol. 115, No. 2, pp. 194-198 (1993).

14. C. H. Suh and C. W. Radcliffe, "Kinematics and Mechanism Design," John Wiley and Sons, New York (1978).

15. G. N. Sandor and A. G. Erdman, "Advanced Mechanism Design: Analysis and Synthesis," Prentice-Hall, Englewood Cliffs (1984).

16. W. Sun and Y. Yuan, "Optimization theory and methods - nonlinear programming", Springer Science+Business Media, New York 